\title{
Histology of the Laennec's Capsule Around the Hepatic Veins and How it May Guide Approaches to Laparoscopic Anatomic Lliver Resection
}

\author{
Kazuteru Monden ${ }^{1}$, Kyotaro Ohno ${ }^{2}$, Hiroshi Sadamori ${ }^{1}$, Masayoshi Hioki ${ }^{1}$, Satoshi Ohno ${ }^{1}$, \\ Norihisa Takakura ${ }^{1}$, Atsushi Sugioka ${ }^{3}$
}

'Department of Surgery, Fukuyama City Hospital, Hiroshima, Japan 2Department of Pathology, Fukuyama City Hospital, Hiroshima, Japan

${ }^{3}$ Department of Surgery, Fujita Health University, Aichi, Japan

\section{ABSTRACT}

Laennec's capsule is a proper membrane of the liver, first described by Réné T. H. Laennec in 1802, covering not only the entire surface of the liver but also the intrahepatic parenchyma surrounding the Glissonean pedicles and the hepatic veins (HVs). It is an essential structure for establishing liver resection, especially anatomic liver resection, which involves the isolation of the extrahepatic Glissonean pedicles and the exposure of the main HVs. Regarding the location of the capsule, Laennec and Couinaud described it as adjacent to the confluence of the main HVs. Whereas, Hayashi revealed it to be around the peripheral branches. Recently, Kiguchi first reported that Laennec's capsule consists of two layers and proposed novel surgical procedures using this concept. Yet, its existence is still debated. Herein, we show the precise anatomy and histology of Laennec's capsule around the HVs including its twolayered composition: one derived from the pericardium (the cardiac Laennec's capsule) and the other from the proper membrane (the hepatic Laennec's capsule). Using these findings, we demonstrate three surgical approaches to the main HVs and emphasize the significance of the craniocaudal parenchymal dissection especially for laparoscopic anatomic liver resection.

Key words: laparoscopy, hepatectomy, Carcinoma, Hepatic vein, Laennec's capsule, Glissonean pedicle

\section{INTRODUCTION}

Laparoscopic liver resections are being performed increasingly worldwide because they have advantages such as magnified images and a unique visual field having dorsal and caudal views (1-4), while being minimally invasive. However, performing them remains technically challenging, especially in anatomic liver resection (5), which requires the isolation of the extrahepatic Glissonean pedicles and the exposure of the main hepatic veins (HVs) for an adequate resection that is neither excessive nor insufficient (1). To standardize the procedure, a precise understanding of the liver anatomy is essential.

\author{
Corresponding author: \\ Kazuteru Monden, M.D. \\ Department of Surgery \\ Fukuyama City Hospital \\ 5-23-1 Zao, Fukuyama \\ Hiroshima 721-8511, Japan \\ Tel: +81-84-941-5151 \\ Fax: +81-84-941-5159 \\ E-mail: monden0319@yahoo.co.jp
}

Received: 18.02 .2020

Accepted: 26.03 .2020

Copyright ( Celsius Publishing House www.sgo-iasgo.com 
Sugioka et al. (6) proposed a new concept of the surgical anatomy of the liver based on Laennec's capsule (7) that covers not only the entire surface of the liver including the bare area but also the intrahepatic parenchyma surrounding the Glissonean pedicles and the main HVs. Therefore, an anatomical gap exists between the Glissonean pedicle and Laennec's capsule, which gives a theoretical background for the extrahepatic Glissonean pedicle isolation without parenchymal destruction-first proposed by Couinaud (8) and performed by Takasaki (9). This concept of Laennec's capsule contributes to standardizing the extrahepatic Glissonean pedicle isolation for anatomic liver resection.

On the other hand, exposing the main HVs remains a difficult procedure due to the lack of standardization. Noteworthily, the concept of Laennec's capsule surrounding the HVs would contribute to standardization, although its existence has remained controversial $(10,11)$. However, Laennec (7) and Couinaud $(12,13)$ had already described the capsule at the confluence of the main $\mathrm{HV}$ and Hayashi et al histologically revealed it to be around the peripheral veins (14). Recently, Kiguchi $(15,16)$ first proposed a novel concept that Laennec's capsule around the HVs is composed of two layers; one derived from the pericardium (the cardiac Laennec's capsule) and the other from the proper membrane (the hepatic Laennec's capsule). These two layers fuse and are integrated into the HV wall becoming indistinguishable at a glance $(12,13)$. However, Kiguchi proposed that Laennec's capsule around the HVs can be preserved and the HVs can be safely exposed by holding their strength. Dissecting the parenchyma from the confluence in the craniocaudal direction, selecting the appropriate layer according to the tumor location and controlling the proximity to the main HV is important because it determines the preservation of the HVs. Thus, this concept of Laennec's capsule contributes to standardizing the surgical approaches for anatomic liver resections by safe exposure of the main and the peripheral HVs. Herein, we demonstrate the histological findings of Laennec's capsule surrounding the HVs and the surgical procedures using this concept.

\section{Histology of Laennec's capsule and anatomy around the hepatic veins}

The histology of Laennec's capsule, especially around the HVs, has not been well investigated. In the first place, Laennec stated that the capsule covered around the confluence of the main HV (7). Couinaud also reported that Laennec's capsule surrounds the main hepatic vein trunk, which immediately fuses with the HV wall becoming indistinguishable $(12,13)$. Both authors described the presence of Laennec's capsule around the confluence of the main hepatic veins but did not mention the covering around the other parts of the HVs, which created confusion for anatomical interpretation $(10,11)$. However, Hayashi et al. (14) clearly demonstrated that Laennec's capsule extends to the peripheral HVs by elastic fiber staining and immunohistochemistry on the liver samples obtained from cadavers.

Our own histological findings in specimens obtained from a patient who underwent right hemi-hepatectomy are shown in figure 1. The liver specimens were vertically sliced into sections along the longitudinal axis of the right HV (RHV) (figure 1a).

The Elastica-van Gieson staining showed that the wavy elastic fibers represent the Laennec's capsule around the wall of the RHV trunk (figure 1b). A peripheral cross-section of the RHV also demonstrates similar wavy elastic fibers (figure 1c). As shown, Laennec's capsule extends to the peripheral RHV branches without covering the central and interlobular veins (figure $1 d$ ), suggesting that these veins have an embryologically different origin.

The liver hanging maneuver introduced by Belghiti et al. (17) is another example that elucidates the capsule's structure around the main HVs and the IVC. During this procedure, a clamp should be passed through the space between the IVC and the liver from the caudal side and the tip of a clamp apparently penetrates the cardiac Laennec's capsule between the middle and RHV, instead of the hepatic one (figures $2 a$ and $b$ ). Histologically, the cardiac Laennec's capsule between the middle and RHV is composed of a mixture of collagen and elastic fibers (figure $2 c$ ). The hepatic one has a similar composition, which indicates that the Laennec's capsule exists around the HVs and consisted of the two layers (figures $1 b$ to $d$ ).

\section{SURGICAL TECHNIQUES}

Laparoscopic central hepatectomies such as right anterior sectionectomy, central bisectionectomy, and segmentectomy 8 are technically demanding because of their location and relationship with the main HVs $(18,19)$. For safely exposing the HVs, the understanding of Laennec's capsule by the surgeon is crucial. First, the surgeons should select the appropriate layer to be exposed near the HV. Although, to hold the strength of the HVs, the full thickness of the Laennec's capsule around the main HVs should be preserved-the 




Figure 1 - The histological findings from the liver specimen obtained by right hemi-hepatectomy.

a. Macroscopic view of the vertical section along the longitudinal axis of the RHV. Each square represents the origin of the histological specimen.

(b)Histological finding of the trunk shows a wavy elastic lamina surrounding the RHV representing Laennec's capsule (Elastica-van Gieson stain, 200X magnified view).

(c) Histological finding of the periphery shows a similar finding of Laennec's capsule surrounding the RHV (Elastica-van Gieson stain, 200X magnified view).

d. Histological finding of the peripheral branch also shows Laennec's capsule surrounding the RHV except for the central and interlobular branches (arrow) (Elastica-van Gieson stain, 100X magnified view). RHV, right hepatic vein.
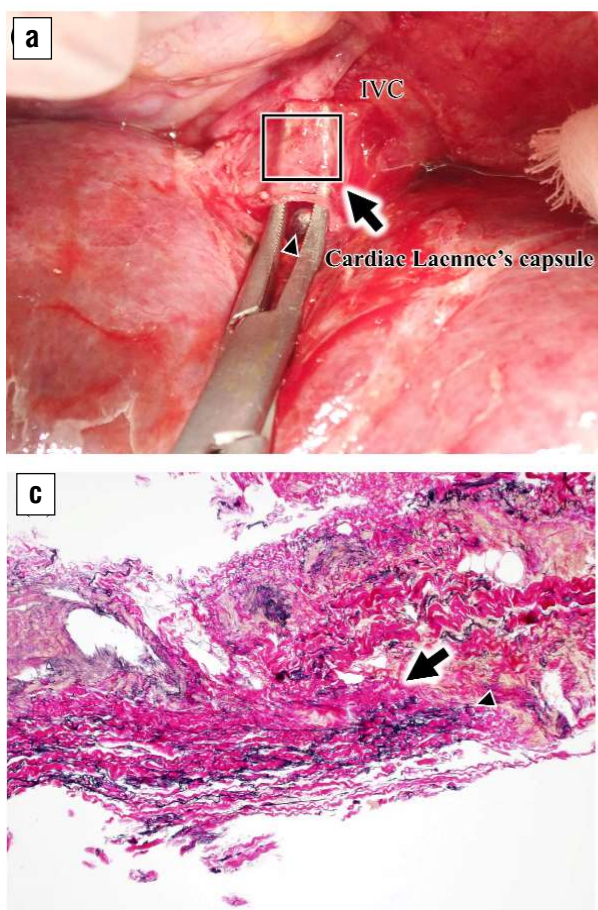

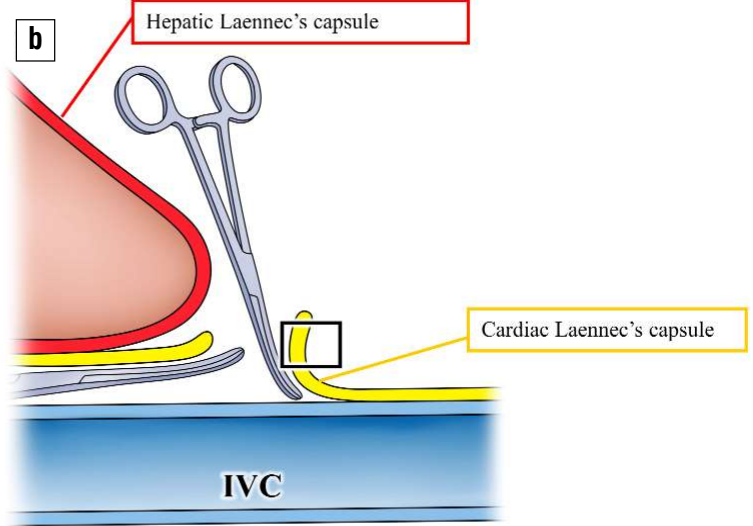

Figure 2 - The cardiac Laennec's capsule demonstrated by liver hanging maneuver.

a. Intraoperative photograph during the liver hanging maneuver, the tip of the clamp (arrowhead) passed underneath the cardiac Laennec's capsule (arrow) and the other clamp isolated it in a caudocranial direction guided by the first clamp.

b. Schema of the cardiac Laennec's capsule (yellow line). A clamp was passed below the cardiac Laennec's capsule guided by penetration of the other clamp.

c. Microscopic image of the squared area showing collagen fibers (arrow) and elastic fibers (arrowhead) representing Laennec's capsule (Elastica-van Gieson stain, $100 \mathrm{X}$ magnified view of the black square area in (a) and (b)). IVC, inferior vena cava. 
outer-Laennec approach-which is preferred. Alternatively, the surgical margin can be ensured by entering the space between the hepatic and cardiac Laennec's capsule-the inter-Laennec approachwhile leaving the hepatic one on the side of the tumor when it is adjacent to the main HVs. Secondly, the parenchymal dissection direction is significant; it should start from the confluence of the main $\mathrm{HV}$ and proceed in the craniocaudal direction to expose the main $\mathrm{HV}$ safely. An advantage of exposing the main HVs from the confluence is that split injury, which is uncontrollable bleeding from the HVs, can be avoided (1). Split injury is caused by moving the dissection device from the peripheral end of the HVs toward the confluence of the HVs. Furthermore, the Glissonean branch is rarely encountered, since the cutting plane is an intersegmental plane during anatomic liver resection. However, these approaches should be supervised by experienced surgeons since the skill of controlling bleeding from the major hepatic veins is essential. In this section, surgical approaches to the main HVs with both layers of Laennec's capsule are described with video.

\section{The Outer-Laennec approach}

In the outer-Laennec approach, the liver parenchyma is dissected preserving both layers of the Laennec's capsule around the HVs (figure 3). First, the liver parenchymal dissection was started just above the main $\mathrm{HV}$ confluence. Then, the liver transection proceeded in the craniocaudal direction that facilitates the preservation of both layers of Laennec's capsule around the HVs. Whereas, if a dissecting device is moved in the caudocranial direction, the capsule might be easily peeled off, which is the inner-Laennec approach.

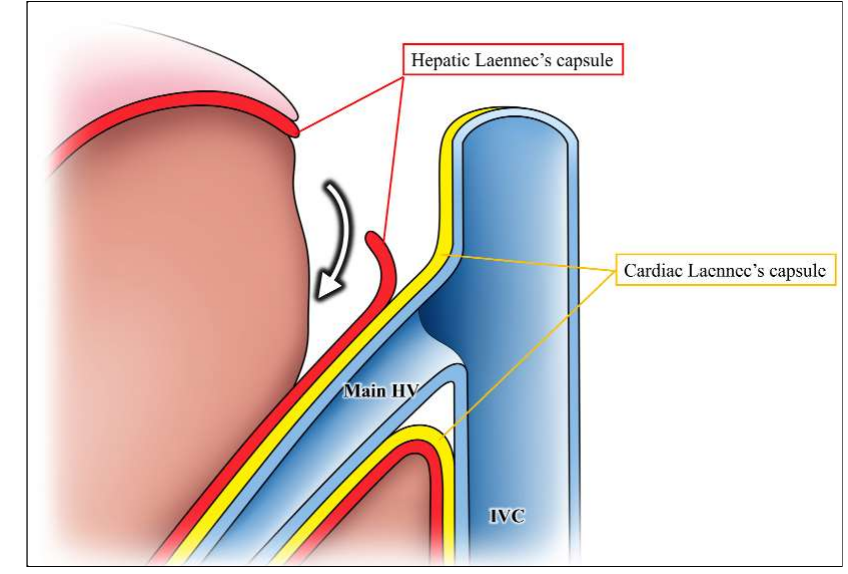

Figure 3 - The outer-Laennec approach for exposure of the main hepatic vein. The outer-Laennec approach can hold the strength of the HV wall because the cardiac and hepatic Laennec's capsules are preserved. IVC: inferior vena cava; HV: hepatic vein

\section{The Inter-Laennec approach}

Kiguchi et al. $(15,16)$ reported that it is possible to enter the layer between the hepatic and cardiac Laennec's capsules to expose the HVs safely, especially in right posterior sectionectomy and segmentectomy 7 . The inter-Laennec approach enables liver dissection preserving only the cardiac Laennec's capsule around the vein wall (figure 4a). This approach is indicated when a tumor is adjacent to the main HVs. The space between the two layers is entered carefully such that it leaves the hepatic Laennec's capsule on the excision side of the liver parenchyma without exposing the tumor surface (figure 4b). The hepatic Laennec's capsule could be confirmed as a shiny membrane covering the cut surface. The preserved cardiac

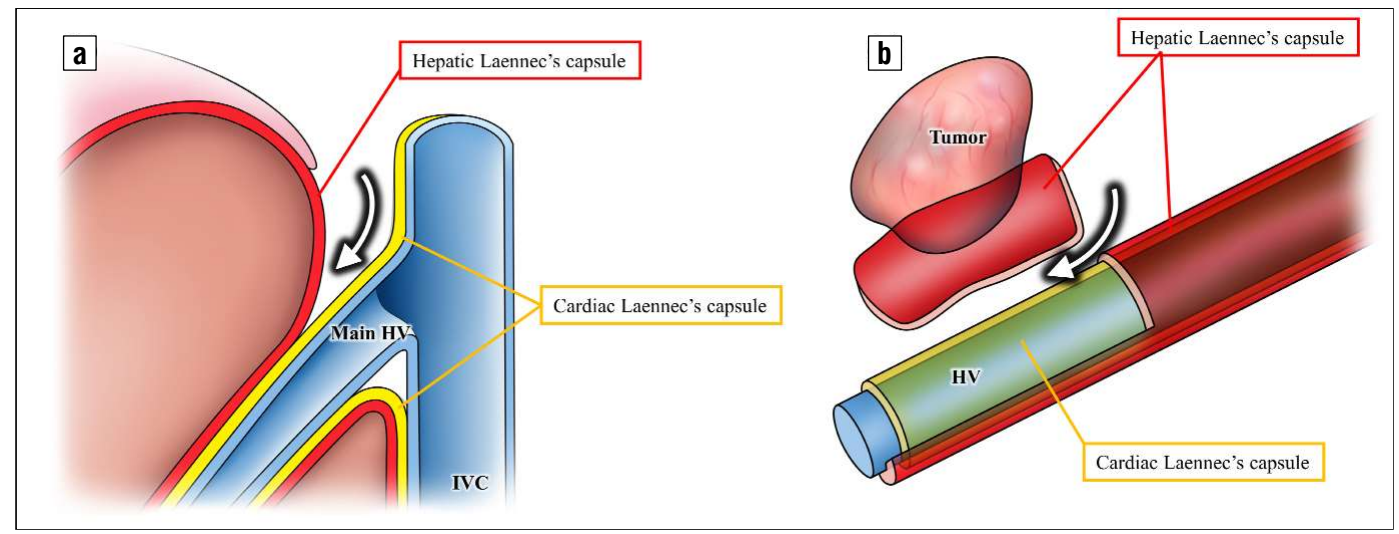

Figure 4 - The inter-Laennec approach for exposure of the main hepatic vein

a. The inter-Laennec approach preserves the cardiac Laennec's capsule around the HV wall holding its strength. b. When a tumor is adjacent to the main HV, the hepatic Laennec's capsule serves to secure the surgical margin. HV: hepatic vein 




Figure 5 - The inner-Laennec approach during liver resection This approach involves peeling off the both the cardiac and hepatic Laennec's capsule. Since the HV wall becomes thin and fragile, this approach should be avoided as possible. When performing conventional liver resection in the caudocranial direction, the transection plane tends to penetrate beneath both the layers; therefore, liver resection in the craniocaudal direction is important for preventing the critical injury to the HVs.

Laennec's capsule serves to hold the strength of the HVs. The inter-Laennec approach facilitates safe anatomic liver resection, especially when a tumor is adjacent to the main HVs.

\section{Video clips for inter- and outer-Laennec approaches}

\section{The Inner-Laennec approach}

The inner-Laennec approach means that the dissection plane enters beneath the two-layered Laennec's capsule while exposing the adventitia of the HV during the parenchymal dissection in the caudocranial direction (figure 5) thinning the HV wall, which makes it fragile. This conventional approach is prone to high bleeding rates. Therefore, we recommend to apply this approach rarely.

\section{CONCLUSION}

The histological findings revealed the Laennec's capsule, which surround the hepatic veins extending over the entire length towards the periphery and having a two layered composition. These findings are essential for the safe exposure of the main hepatic veins and standardizing the anatomic liver resection by approaching the appropriate layer of Laennec's capsule.

\section{Conflicts of Interest and Source of Funding}

None declared. This study received no specific grant from any funding agency.

\section{REFERENCES}

1. Honda G, Kurata M, Okuda Y, Kobayashi S, Tadano S, Yamaguchi T, et al. Totally laparoscopic hepatectomy exposing the major vessels. J Hepatobiliary Pancreat Sci. 2013;20(4):435-40.

2. Iwashita $\mathrm{Y}$, Uchida $\mathrm{H}$, Endo $\mathrm{Y}$, Yada $\mathrm{K}$, Mori $\mathrm{T}$, Ohta $\mathrm{M}$, et al. Laparoscopic caudal approach for a small hepatocellular carcinoma located in the paracaval portion of a cirrhotic liver. Asian J Endosc Surg. 2016;9(3):204-7.

3. Wakabayashi G, Cherqui D, Geller DA, Buell JF, Kaneko H, Han HS, et al. Recommendations for laparoscopic liver resection: a report from the second international consensus conference held in Morioka. Ann Surg. 2015;261(4):619-29.

4. Nguyen KT, Gamblin TC, Geller DA. World review of laparoscopic liver resection-2,804 Patients. Ann Surg. 2009;250(5):831-41.

5. Ban D, Tanabe M, Ito H, Otsuka Y, Nitta H, Abe Y, et al. A novel difficulty scoring system for laparoscopic liver resection. J Hepatobiliary Pancreat Sci. 2014;21(10):745-53.

6. Sugioka A, Kato $Y$, Tanahashi $Y$. Systematic extrahepatic Glissonean pedicle isolation for anatomical liver resection based on Laennec's capsule: proposal of a novel comprehensive surgical anatomy of the liver. J Hepatobiliary Pancreat Sci. 2017;24(1):17-23.

7. RTH Laennec. Lettre sur des Tuniques qui enveloppent certains Viscères, et fournissentdes gaines membraneuses à leurs vaisseaux. Journ De Méd Chir Et Pharm Vendémiaire an XI; (1802) 539-575, et Germinal an Xl; (1802) 73-89.

8. Couinaud C. A simplified method for controlled left hepatectomy. Surgery 1985; 97:358-61.

9. Takasaki K, Kobayashi S, Tanaka S. Muto H, Watayo T, Saito A, et al. Newly developed systematized hepatectomy by Glissonean pedicle transection method. Syujutsu. 1986; 40:7-14. (in Japanese)

10. Shirata C, Hasegawa K, Halkic N, Kokudo N. Laennec's capsule does not exist around the peripheral hepatic veins. J Hepatobiliary Pancreat Sci. 2019; 26(10):E13.

11. Sugioka A. Re: Laennec's capsule does not exist around the peripheral hepatic veins. J Hepatobiliary Pancreat Sci. 2019; 26(10):E14.

12. Couinaud C. Le foie; études anatomiques et chirurgicales. Paris: Masson; 1957:221-222.

13. Couinaud C. The vasculo-biliary sheath. Surgical anatomy of the liver revisited. Paris: pers Ed; 1989. p. 29-39.

14. Hayashi S, Murakami G, Ohtsuka A, Itoh M, Nakano T, Fukuzawa Y. Connective tissue configuration in the human liver hilar region with special reference to the liver capsule and vascular sheath. J Hepatobiliary Pancreat Surg. 2008:15(6):640-7.

15. Kiguchi G, Sugioka A, Kato Y, Uyama I. Use of the inter-Laennec approach for laparoscopic anatomical right posterior sectionectomy in semi-prone position. Surg Oncol. 2019; 29:140-1.

16. Kiguchi G, Sugioka A, Kato $Y$, Uyama I. Laparoscopic S7 Segmentectomy using the inter-Laennec approach for hepatocellular carcinoma near the right hepatic vein. Surg Oncol. 2019; 31:132-4.

17. Belghiti J, Guevara OA, Noun R, Saldinger PF, Kianmanesh R. Liver hanging maneuver: A safe approach to right hepatectomy without liver mobilization. J Am Coll Surg 2001;193(1):109-11.

18. Chanwat R, Bunchaliew C, Tanompetsanga R, Orannapalai N. How I do it: laparoscopic anatomical segment 8 resection. Ann Laparosc Endosc Surg. 2018; 3:58.

19. Ome Y, Honda G, Doi M, Muto J, Seyama Y. Laparoscopic anatomic liver resection of segment 8 using intrahepatic Glissonean approach. J Am Coll Surg 2019. pii: S1072-7515(19)32221 